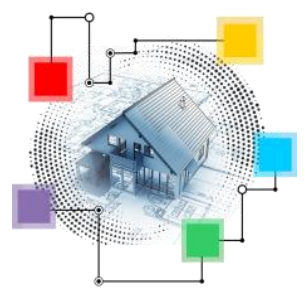

\author{
XIII SIMPÓSIO NACIONAL DE SISTEMAS PREDIAIS \\ DESEMPENHO E INOVAÇÃO \\ DE SISTEMAS PREDIAIS HIDRÁULICOS \\ SÃO PAULO - 04 DE OUTURO DE 2019
}

\title{
Caracterização dos usos-finais de água de edifícios comerciais: estudo de caso de um café em Brasília-DF
}

\section{Characterization of the water end uses of commercial buildings: case study of a coffee shop in Brasília, Brazil}

\author{
TOTUGUI, Natália'; SANT'ANA, Daniel2; SANTOS, Susanna ${ }^{3}$; SANTANA, Livia ${ }^{4}$
}

\footnotetext{
${ }^{1}$ Grupo de Pesquisa Água \& Ambiente Construído, Universidade de Brasília, nataliatotugui@gmail.com

${ }^{2}$ Grupo de Pesquisa Água \& Ambiente Construído, Universidade de Brasília, dsantana@unb.br

${ }^{3}$ Grupo de Pesquisa Água \& Ambiente Construído, Universidade de Brasília, susannasantos1@gmail.com

${ }^{4}$ Grupo de Pesquisa Água \& Ambiente Construído, Instituto Federal de Goiás, livia.santana@ifg.edu.br
}

\begin{abstract}
RESUMO
O Distrito Federal enfrentou estresse hídrico em 2016, com baixa disponibilidade hídrica para abastecimento urbano. A fim de reduzir a demanda de água urbana e promover a conservação da água, é crucial entender como a água está sendo usada em diferentes tipos de edifícios. Este artigo tem como objetivo caracterizar e analisar os usos-finais de água de um café localizado em um edifício comercial na Asa Norte, Brasília. Para tanto, foram adquiridas as contas de água mensais do café, realizada uma vistoria técnica para identificar as diferentes composições hidráulicas das instalações no estabelecimento e, em seguida, foi efetuada uma auditoria hidráulica para caracterizar os usos-finais de água. A auditoria do consumo contou com instalações de equipamentos de medição data-loggers em todos os pontos hidráulicos. Constatou-se que esse tipo de atividade comercial tem maior consumo no setor de cozinha, onde o uso-final com maior demanda é o da pia de cozinha (54\%), seguido por descarga sanitária (20\%). Conclui-se que os resultados foram bastantes positivos, pois a discrepância entre o valor mensurado pelos data-loggers e o valor médio diário faturado pela CAESB foi de apenas - $11 \%$ do consumo que não pôde ser explicado pelos usos-finais, validando, dessa forma, o método utilizado neste estudo.
\end{abstract}

Palavras-chave: Estabelecimento comercial; Usos-finais de água; Indicadores de consumo de água.

\begin{abstract}
The District Federal faced water stress in 2016, with alarming low levels of water availability for urban water supply. In order to reduce water demand and promote water conservation, it is crucial to understand how water is used in different types of buildings. This study characterized water end-uses of a coffee shop located in a commercial building in Brasilia, Brazil. To do this, this study made use a water auditing technique to characterize the water end uses that made use data-loggers to measure water consumption at points of use. Results suggest that the water demand is highest in the kitchen sink (54\%) followed by toilet flushing (20\%). The discrepancy between measured values from data loggers and average daily metered consumption was $11 \%$.
\end{abstract}

Keywords: Commercial establishments; Water end-uses; Water consumption indicators. 


\section{INTRODUÇÃO}

O Distrito Federal viveu importante exaustão hídrica em 2016, com níveis alarmantes em seus principais reservatórios (ADASA, 2016). Uma vez que os recursos naturais chegam ao seu limite, em função de uma exploração exagerada, torna-se indispensável práticas direcionadas ao controle da demanda de água. A fim de reduzir a sobrecarga de exploração dos recursos hídricos, a aplicação de distintas estratégias conservadoras de água em edificações tem sido vista como uma providência eficaz para a redução da demanda urbana de água (ALVARES e SANT'ANA, 2016).

Nesse cenário, é importante compreender como a água está sendo empregada em diferenciados setores residenciais, comerciais, industriais e institucionais. Isto posto, com o intuito de analisar o desempenho de diferentes métodos conservadores de água e verificar soluções viáveis voltadas à redução do consumo de água nas edificações é essencial caracterizar o consumo de água em seus usos-finais e compreender os hábitos de consumo dos usuários (DE OREO et al., 1996). Alguns estudos internacionais caracterizaram os usos-finais de água em edifícios comerciais e públicos (DZIEGIELEWSKI et al., 2000; FARINA et al., 2011; SURRENDRAN, S. e WHEATLEY, 1998; WAGGETT e AROTSKY, 2006). Os estudos sobre usos-finais do consumo em edificações não-residenciais propiciam pesquisas sobre o potencial de redução da demanda para uma variedade de técnicas conservadoras e seus custos-benefícios (GRIGGS et al., 1998; PASCHKE et al.,2002; MADDAUS e MADDAUS, 2004). Todavia, no Brasil, as descrições dos usos-finais do consumo de água são apenas primícias.

Até onde vai a literatura, a análise dos usos-finais de água em edifícios não-residenciais no Brasil resume-se a edifícios públicos (KAMMERS e GHISI, 2006), escritórios em Florianópolis (PROENÇA e GHISI, 2010), escolas (FASOLA et al., 2011; MARINOSKI e GHISI, 2008; SILVA et al., 2006) e hotéis em Brasília (SANT'ANA e NASCIMENTO, 2014). Outras pesquisas nacionais sobre conservação de água em edificações não residenciais identificaram o potencial de redução do consumo de água em hospitais (ILHA et al., 2006), indústria (MIERZWA e HESPANHOL, 2005) e instituições de ensino (MEDEIROS et al., 2012; FASOLA et al., 2011; MARINOSKI e GHISI, 2008; SILVA et al., 2006). No que se diz respeito a edificações comerciais, estudos limitam-se a padarias em São Paulo (MOTTA e SANCHEZ, 2001) e Juazeiro (GOMEZ e ALVEZ, 2000), posto de gasolina em Brasília (GHISI et al., 2009) e shopping center no Rio de Janeiro (NUNES, 2006).

Observa-se que há uma carência de dados quando se refere aos usos-finais do consumo de água em diversas atividades relacionados ao comércio. Torna-se necessária a ampliação de estudos que quantifiquem os usos-finais de água e os hábitos de consumo no setor comercial para, posteriormente, promover a conservação de água no comércio, preservando os recursos hídricos locais e contribuindo com um desenvolvimento sustentável. Dessa forma, este artigo tem como objetivo caracterizar e analisar os usos-finais de água de um café localizado em um bloco comercial na 407 norte, Asa Norte, Brasília, Distrito Federal. 


\section{MÉTODO}

Para o cumprimento dos objetivos propostos os procedimentos metodológicos foram divididos nas três seguintes etapas: i) entrevistas estruturadas e visita técnica; ii) obtenção de contas de água; e iii) auditoria hidráulica.

Como ponto de partida, foram efetuadas entrevistas com questionários estruturados com o gerente e funcionários do café, nesse mesmo dia, foi realizada a vistoria técnica no estabelecimento. Por meio dos questionários, foram obtidos dados relativos aos horários de funcionamento da loja, área construída, número da população fixa (funcionários), número médio da população flutuante (clientes), lavagem de piso (frequência e modo de lavagem) e hábitos de consumo dos funcionários. Já a vistoria técnica, utilizada como preparação para a auditoria do consumo, teve como objetivo identificar todos os pontos dos usos-finais de água, registrar as características de todos os equipamentos hidrossanitários e avaliar as condições da rede de distribuição, a fim de detectar eventuais vazamentos. Dessa forma, foi possível quantificar o número e os tipos de equipamentos que precisariam para instalar os data-loggers, na etapa da auditoria hidráulica, para mensurar os usos-finais de todo o estabelecimento comercial.

Em seguida, foram adquiridas as contas de água mensais do estabelecimento comercial, por meio da Companhia de Saneamento Ambiental do Distrito Federal (CAESB), relativas ao período de janeiro de 2013 a dezembro de 2017. Por meio desses dados, foi possível obter a média de consumo mensal.

A terceira etapa trata de técnicas de auditoria de consumo de água, que contou com instalações de equipamentos de medição data-loggers em todos os pontos hidráulicos, para registrar eventos de consumo com o intuito de medir o consumo de cada uso-final de água do café analisado. Foram utilizados diários de registros ou registros fotográficos, questionários e observações in-loco para caracterizar os usos-finais de água do estabelecimento. Apesar das diferentes técnicas, a base da auditoria hidráulica contou com a instalação de equipamentos de medição data-loggers em pontos de uso para registrar eventos de consumo de água (Erro! Fonte de referência não encontrada.). Demais técnicas foram utilizadas com o intuito de validar/verificar resultados obtidos nas medições, ou como técnica complementar.

FIGURA 1 - Equipamento de medição composto por medidor de fluxo (a) e módulo data-logger (b).

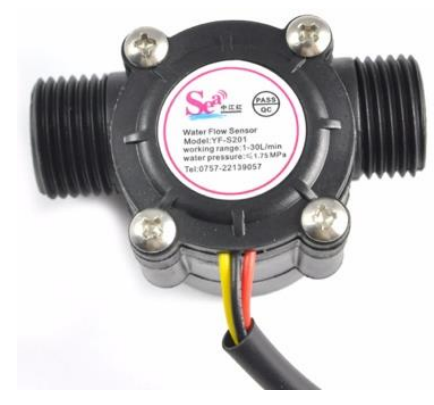

(a)

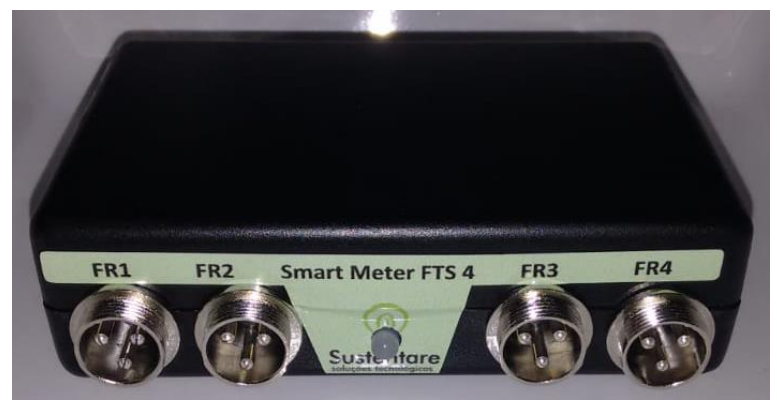

(b)

O equipamento data-logger utilizado é composto por medidores de fluxo tipo turbina de 1/2" capazes de medir o volume de água utilizado entre vazões de $1 \mathrm{l} / \mathrm{min}$ a $30 \mathrm{l} / \mathrm{min}$. Os medidores 
de fluxo possuem um sensor que emite um pulso a cada giro de dente da turbina, encaminhando, por meio de um cabo de sinal blindado, as informações do evento de uso de água a um módulo data-logger auxiliar que computa sua velocidade de rotação e tempo e, por conseguinte, armazena o volume utilizado em um cartão de memória SD.

\section{RESULTADOS E DISCUSSÃO}

O café selecionado para estudo de caso, situado em Brasília, oferece bebidas quentes e geladas, salgados e lanches feitos na hora. Funciona segunda a sexta-feira de 7 h30 às 20h, sábado de $8 \mathrm{~h} 30$ às $16 \mathrm{~h}$ e fecha aos domingos. Esse estabelecimento possui $70 \mathrm{~m}^{2}$ de área construída, 7 funcionários e atende cerca de 100 pessoas por dia. O consumo de água médio é de $286 \mathrm{~m}^{3} / \mathrm{ano}$ (784 l/dia), ou seja, o consumo per area é de $11,2 \mathrm{l} / \mathrm{m}^{2} /$ dia e o consumo per capita, de 8 l/pes./dia.

FIGURA 2 - Café selecionado para estudo de caso
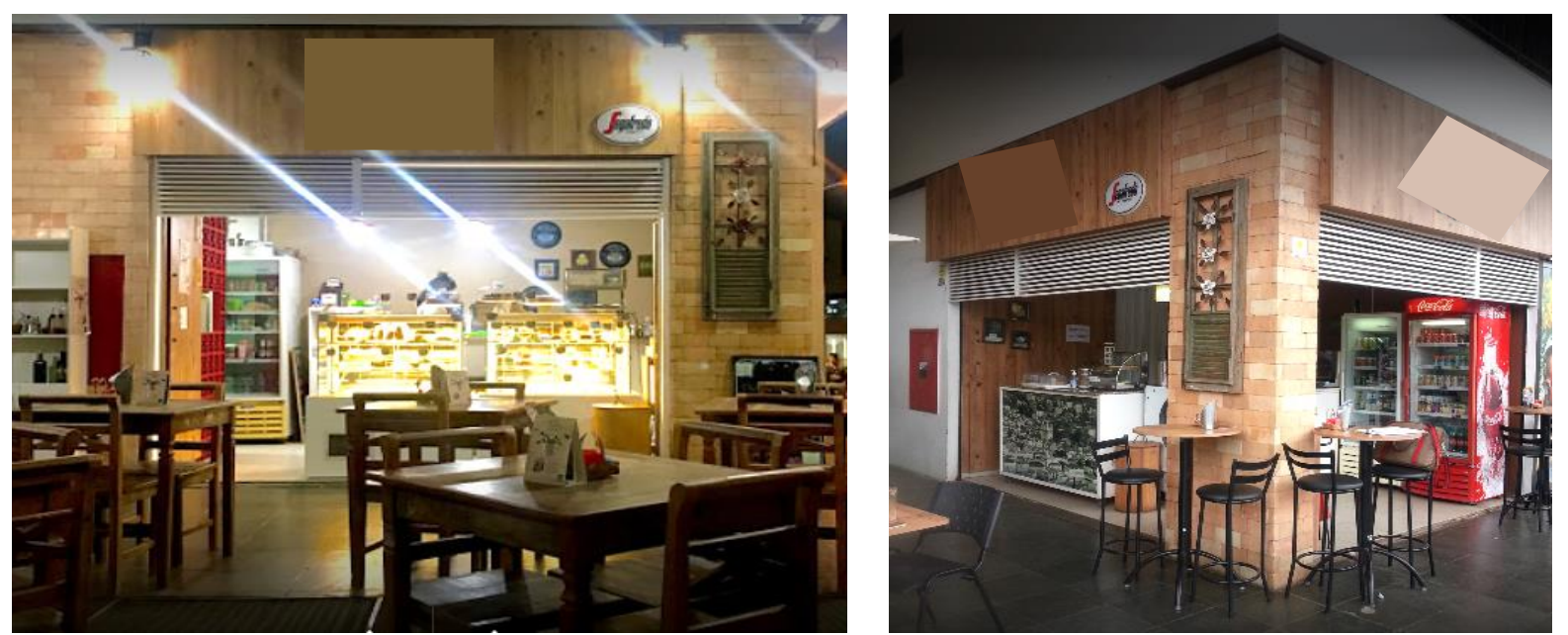

Fonte: Google Earth (2019)

Esse estabelecimento possui três ambientes com pontos de água, um banheiro misto para clientes, uma cozinha e o balcão de preparo do café. As informações coletadas para cada aparelho hidráulico instalado por ambiente constam na TABELA 1 abaixo. As vazões obtidas pelos equipamentos foram conferidas com as vazões mensuradas no local e estão com resultados muito próximos, validando os métodos utilizados para essa coleta. A partir dos dados de tempo de uso, da vazão, e da frequência foi possível identificar o consumo diário em litro por dia para cada ambiente, e os indicadores de consumo por uso-final. A torneira de uso geral é utilizada apenas para a limpeza dos pisos da loja, por isso seu indicador é em litro por área por dia $\left(\mathrm{l} / \mathrm{m}^{2} /\right.$ dia $)$. 
TABELA 1 - Vazão, tempo, frequência, consumo e indicador por uso-final de água

\begin{tabular}{|c|c|c|c|c|c|}
\hline Uso Final & Vazão & $\begin{array}{c}\text { Tempo } \\
(s)\end{array}$ & $\begin{array}{c}\text { Freq } \\
\text { (n/dia) }\end{array}$ & $\begin{array}{l}\text { Consumo } \\
\text { (l/dia) }\end{array}$ & $\begin{array}{l}\text { Indicador } \\
\text { (l/pes./dia) }\end{array}$ \\
\hline Banheiro & & & & & 21,4 \\
\hline Lavatório & $0,07 \mathrm{l} / \mathrm{s}$ & 9 & 21 & 13 & 1,8 \\
\hline Descarga Sanitária & $6,00 l p f$ & 40 & 1 & 138 & 19,6 \\
\hline Café & & & & & 1,6 \\
\hline Máq. Expresso & $0,01 \mathrm{l} / \mathrm{s}$ & 5 & 39 & 11 & 1,6 \\
\hline Cozinha & & & & & 76,2 \\
\hline Lavatório & 0,05 & 9 & 120 & 52 & 7,4 \\
\hline Pia de Cozinha & $0,04 \mathrm{l} / \mathrm{s}$ & 12 & 370 & 186 & 26,6 \\
\hline Pia de Cozinha & 0,04 & 12 & 377 & 192 & 27,5 \\
\hline Máq. Lavar Louças & $0,04 \mathrm{l} / \mathrm{s}$ & 12 & 203 & 103 & 14,7 \\
\hline
\end{tabular}

$l / s=$ litro por segundo $;$ lpf = litro por fluxo; $n /$ dia = número por dia $;$ l/dia = litro por dia l/pes./dia = litro por pessoa por dia $; \mathrm{l} / \mathrm{m}^{2} / d=$ litro por metro quadrado dia

A proporção do consumo nos usos-finais analisados do café, conforme pode ser observado na FIGURA 3, aponta que grande parte do consumo de água desse tipo de estabelecimento comercial é no setor de cozinha. No qual, o uso-final com maior demanda é o da pia de cozinha (54\%), seguido por descarga sanitária (20\%), máquina de lavar louça (15\%), lavatório (9\%) e máquina de café expresso (2\%).

\section{FIGURA 3 - Consumo nos usos finais}

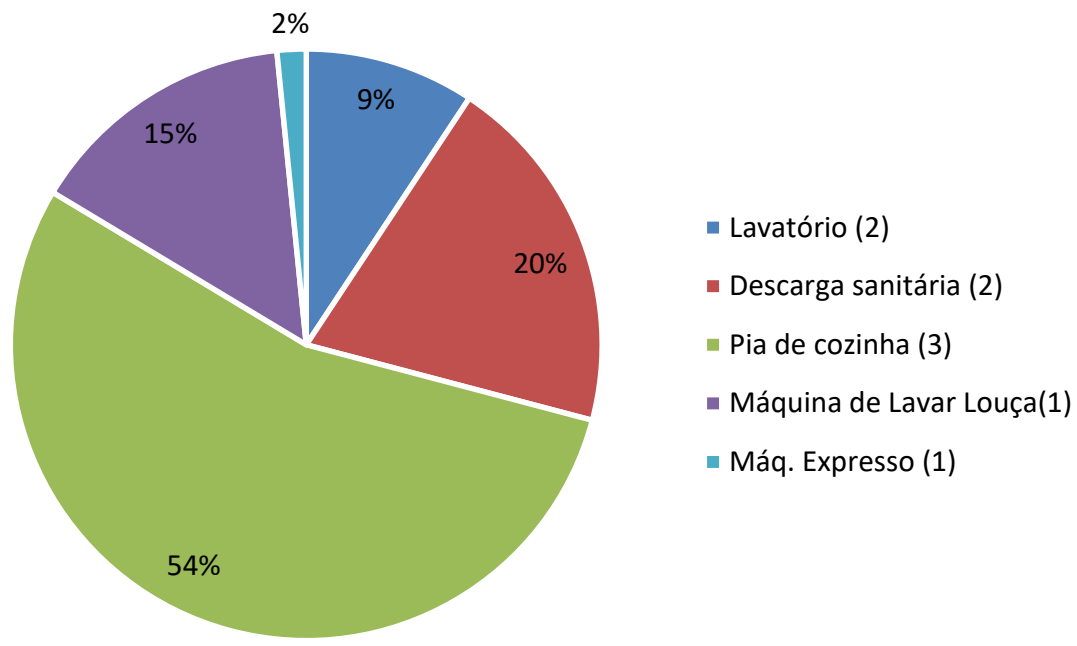

Por fim, com os resultados obtidos em cada uso-final foi estimado o consumo diário do café e comparados com o consumo médio diário faturado pela CAESB. Este, dado pela somatória do consumo anual em litros, do ano de 2017, dividido por 365 dias. Ao fazer o cálculo de discrepância entre esses valores verificou-se que aproximadamente $11 \%$ do consumo não pôde ser explicado pelos usos-finais. Esse resultado é bastante positivo porque ao longo do ano 
podem ter havido intercorrências como variação de número de clientes de um mês a outro, feriados, hábitos de consumo que acabam variando um pouco, economia hídrica, entre outros. Todavia, mesmo assim, os resultados obtidos dos data-loggers estão bastante próximos.

TABELA 2 - Uso-final

\begin{tabular}{lc}
\hline Usos-Final de Água & Consumo (l/dia) \\
\hline Lavatório $(\mathrm{n}=2)$ & 65 \\
Descarga sanitária $(\mathrm{n}=2)$ & 138 \\
Pia de cozinha $(\mathrm{n}=3)$ & 378 \\
Máquina de Lavar Louça( $\mathrm{n}=1)$ & 103 \\
Máq. Expresso $(\mathrm{n}=1)$ & 11 \\
Total Estimado & $\mathbf{6 9 4}$ \\
Média Faturada & $\mathbf{7 8 4}$ \\
Discrepância & $\mathbf{- 1 1 , 4 1 \%}$ \\
\hline \hline
\end{tabular}

\section{CONSIDERAÇÕES FINAIS}

Este artigo caracterizou os usos-finais de água de um café situado na 407 Norte, Asa Norte, Brasília, Distrito Federal. Para tal, o estudo realizou auditoria hidráulica contando com a instalação de medidores de fluxo e equipamentos data-loggers em todos os pontos hidráulicos do estabelecimento, permanecendo instalado por sete dias consecutivos. As vazões obtidas pelos equipamentos foram conferidas com as vazões mensuradas no local e estão com resultados muito próximos, validando os métodos utilizados para essa coleta. Ainda, a discrepância entre o valor mensurado pelos data-loggers e o valor médio diário faturado pela companhia de abastecimento foi de apenas $-11 \%$ do consumo que não pôde ser explicado pelos usos-finais. Resultado que foi muito favorável, pois ao longo do ano podem ter ocorrido intercorrências influenciando o consumo para mais ou para menos.

Verificou-se que esse tipo de atividade, que está diretamente relacionada ao preparo e fornecimento de alimentos tem maior consumo no setor de cozinha. No qual, o uso-final com maior demanda é o da pia de cozinha (54\%), seguido por descarga sanitária (20\%), máquina de lavar louça (15\%), lavatório (9\%) e máquina de café expresso (2\%). O consumo de água médio do café foi de $286 \mathrm{~m}^{3} /$ ano $\left(784 \mathrm{l} / \mathrm{d}\right.$ ) e o consumo per area e per capita foram de $11,2 \mathrm{l} / \mathrm{m}^{2} / \mathrm{dia}$ e de 8 l/pes./dia, respectivamente.

\section{AGRADECIMENTOS}

Os autores agradecem o financiamento desta pesquisa cedido pela Agência Reguladora de Águas, Energia e Saneamento Básico do Distrito Federal (ADASA), a Companhia de Saneamento Ambiental do Distrito Federal (CAESB) por sua prestimosa colaboração com o estudo, providenciando as informações necessárias para o desenvolvimento do trabalho e aos demais colaboradores que contribuíram com o estudo. 


\section{REFERÊNCIAS}

ADASA, Agência Reguladora de Águas, Energia e Saneamento Básico no Distrito Federal. Níveis dos Reservatórios 11/2016. 09 nov. 2016. Disponível em: http://www.adasa.df.gov.br/monitoramento/niveis-dos reservatorios/monitoramento/niveis-dosreservatorios/671-nivel-dos-reservatorios-11-2016> Acesso em: 06 abril 2019.

ALVARES, Karla; SANT'ANA, Daniel. Reduções na exploração de recursos hídricos pelo emprego de tecnologias conservadoras de água: uma análise piloto em regiões residenciais do Distrito Federal: Desafios e Perspectivas da Internacionalização da Construção. In: ENTAC, 16., 2016, São Paulo. Anais... . Porto Alegre: ANTAC, 2016, p. 4372 - 4385. Disponível em: <http://www.infohab.org.br/entac/2016/ENTAC2016_paper_696.pdf>. Acesso em: 03 fev. 2018.

DEOREO, W.B., HEANEY, J.P., MAYER, P.W. Flow trace analysis to assess water use. Journal of American Water Works Association, V.88, nº 1, p. 79 90, jan., 1996.

DZIEGIELEWSKI, B. et al. Commercial and institutional end uses of water. Denver: AWWA Research Foundation, 2000. 264p.

FARINA, M.; MAGLIONICO, M.; POLLASTRI, M.; STOJKOV, I. Water consumption in public schools. Procedia Engineering, v.21, p.929-938, 2011.

FASOLA, B. G.; GHISI, E.; MARANOSKI, K. A.; BORINELLI, B. J. Potencial de economia de água em duas escolas em Florianópolis, SC. Ambiente Construído, Porto Alegre, v. 11, n. 4, p. 6578, out./dez. 2011.

GHISI, E., TAVARES, D. F., ROCHA, V. L. Rainwater harvesting in petrol stations in Brasília: Potential for potable water savings and investment feasibility analysis. Resources, Conservation and Recycling, v.54, n.2, p.79-85, 2009.

GOMEZ, J.; ALVES, W. Final water consumption in building installations using the flow-rate trace. Proceedings $\mathbf{2 6}^{\text {th }}$ International Symposium on Water Supply and Drainage for Buildings. Rio de Janeiro: USP, 2000.

GRIGGS, J. C.; SHOULER, M. C.; HALL, J. Water conservation and the built environment. In: 21 AD: Water. Oxford: Oxford University Press. 1998.

ILHA, M. S. O.; NUNES, Solange da Silva; SALERMO, Lia Soares . Programa de conservação de água em hospitais: estudo de caso do Hospital das Clínicas da Universidade Estadual de Campinas. Ambiente Construído (São Paulo. Impresso), v. 6, n.1, p. 91-97, 2006.

KAMMERS, P. C.; GHISI, E. Usos finais de água em edifícios públicos localizados em Florianópolis, SC. Ambiente Construído, Porto Alegre, v.6, n.1, p.75-90, 2006.

MADDAUS, W.O.; MADDAUS, M. L. Evaluating water conservation cost-effectiveness with an enduse model. Proceedings Water Sources Conference, Austin Texas: AWWA, 2004.

MARINOSKI, K. A.; GHISI, E. Aproveitamento de água pluvial para usos não potáveis em instituição de ensino: estudo de caso em Florianópolis - SC. Ambiente Construído, Porto Alegre, v. 8, n. 2, p. 67-84, abr./jun. 2008.

MEDEIROS, Gerson Araujo de; CARVALHO JUNIOR, Osvaldo Oliveira de; VACCARI, Gabrielly Boer. Potencialidades do reuso da água: estudos de caso no setor sucroalcooleiro e universitário. Unipinhal, Espírito Santo do Pinhal, v. 9, n. 1, p.03-16, mar. 2012. 
MIERZWA, José Carlos; HESPANHOL, Ivanildo. Água na Indústria: uso racional e reúso. São Paulo: Oficina de Textos, 2005. 144 p.

MOTTA, Sandro de Almeida; SANCHEZ, Jorge Gomez. Diagnóstico e parametrização do consumo de água em padarias da RMSP. In: CONGRESSO BRASILEIRO DE ENGENHARIA

SANITÁRIA E AMBIENTAL, 21., 2001, Rio de Janeiro. Anais do $21^{\circ}$ Congresso Brasileiro de Engenharia Sanitária e Ambiental. Rio de Janeiro: Abas, 2001. p. 1 - 13. Disponível em:

$<$ http://docplayer.com.br/16084659-I-036-diagnostico-e-parametrizacao-do-consumode-agua-empadarias-da-rmsp.html>. Acesso em: 20 maio 2018.

NUNES, Riane Torres Santiago. Conservação da água em edifícios comerciais: Potencial de uso racional e reúso em Shopping Center. 2006. 144 f. Dissertação (Mestrado) - Curso de Engenharia, Ciências em Planejamento Energético, Universidade Federal do Rio de Janeiro, Rio de Janeiro, 2006.

PASCHKE, P.; VAN GELDER, R. E.; SIEGELBAUM, H. Hotel water conservation: A Seattle demonstration. Seattle: Seattle Public Utilities, 2002. 127p.

PROENÇA, L. C.; GHISI, E. Water end-uses in Brazilian Office buildings. Resources, Conservation and Recycling, v.54, n.8, p.489-500, 2010.

SANT'ANA, Daniel; NASCIMENTO, Eduarda Aun de Azevedo. Caracterização dos usos-finais do consumo de água em edificações do Setor Hoteleiro de Brasília. Revista de Arquitetura da Imed, Passo Fundo Rs, v. 3, n. 2, p.156-167, nov. 2014. Semestral.

SILVA, G. S.; TAMAKI, H. O.; GONÇALVES, O. M. Implementação de programas de uso racional da água em campi universitários. Ambiente Construído, Porto Alegre, v.6. n.1, p.49-61, 2006.

SURRENDRAN, S.; WHEATLEY, A.D. Grey-water reclamation for non-potable re-use. Water and Environment Journal. v. 12, n.6, p.406-413. 1998.

WAGGETT, R.; AROTSKY, C. Water key performance indicators and benchmarks for offices and hotels. London: CIRIA, 2006. 56p. 